Cinémas

Revue d'études cinématographiques

Journal of Film Studies

\title{
Inédit : Rayon et Gnôle [Essai de définition de la carence idéologique dans le domaine de la forme]
}

\section{Sergueï M. Eisenstein}

Volume 11, numéro 2-3, printemps 2001

Eisenstein dans le texte

URI : https://id.erudit.org/iderudit/024848ar

DOI : https://doi.org/10.7202/024848ar

Aller au sommaire du numéro

Éditeur(s)

Cinémas

ISSN

1181-6945 (imprimé)

1705-6500 (numérique)

Découvrir la revue

Citer cet article

Eisenstein, S. M. (2001). Inédit : Rayon et Gnôle [Essai de définition de la carence idéologique dans le domaine de la forme]. Cinémas, 11(2-3), 73-109. https://doi.org/10.7202/024848ar d'utilisation que vous pouvez consulter en ligne. 


\section{INÉDIT}

\section{Rayon et Gnôle \\ [Essai de définition \\ de la carence idéologique dans le domaine de la forme ${ }^{1}$ ]}

\section{Sergueï M. Eisenstein}

Jamais, dans aucun travail, je n'ai eu de directeur, politique ou autre. Je travaille comme me le dicte mon sentiment intérieur personnel. Je crois que la confusion des questions de politique avec l'art est quelque chose de très nuisible, tout comme la surveillance.

Extrait du télégramme de I. Perestiani paru dans Kino-nedelja $\mathrm{n}^{\circ} 13^{2}$

Tout en considérant qu'il est indigne de polémiquer avec cette sorte de point de vue "antédiluvien" en l'an 1925, je choisis cette épigraphe monstrueuse, parce que l'analyse proposée va concerner très précisément la question de la nécessité et de la possibilité de surveiller les réalisateurs dans le domaine de ce que leur "dicte le sentiment personnel intérieur".

Étant donné le "type même d'arme", l'utilisation révolutionnaire de spets $^{3}$ dans le domaine des arts ne peut pas se faire selon les mêmes procédés que ceux adoptés pour les spets militaires, techniques, etc. : on institue un "commissaire militaire" et le tour est joué. Parce que dans ces domaines le travail du spets 
consiste pour l'essentiel à appliquer ses savoirs de façon mathématique, et que la part d'initiative personnelle (de talent) n'intervient que dans une moindre mesure. Il s'agit d'une production purement consciente, tant pour le spets (qui l'exécute) que pour le commissaire (qui la surveille) dont le contrôle se borne à établir l'honnêteté technique avec laquelle les tâches sont effectuées par le spets indépendamment de ses aspirations idéologiques intérieures, sa possible opposition intérieure à la révolution, etc. La différence ne tient pas non plus à ce qu'on a affaire dans le cas de l'art à une production idéologique, tandis que dans la guerre, il n'y aurait pas d'idéologie.

Dans le cas des spets du domaine artistique, l'affaire est beaucoup plus complexe et exige qu'on approfondisse substantiellement les questions du "contrôle".

Je crois catégoriquement erronée l'opinion partagée, malheureusement, par la majorité des camarades du Parti, selon laquelle on pourrait, ici aussi, se limiter à l'honnêteté technique - en servant ledit contenu le mieux possible. Selon laquelle l'évaluation de l'aspect formel de ce genre de travail se réduirait finalement à cela. Dans les questions d'idéologie, l'aspect formel n'est pas moins indépendant et autodéterminant que le "contenu».

Aussi correct que soit le contenu, le rôle corrupteur d'une forme déterminée par une autre appartenance de classe, par d'autres principes, est un phénomène non seulement parfaitement imaginable, mais étayé par un certain nombre de faits précis.

Qu'est-ce que le contenu?

Prenons un exemple au hasard: La Fin de l'espoir ${ }^{4}$, œuvre idéologiquement irréprochable, "recommandée" (bien que même sous cet aspect - celui du contenu, de sa mise en forme dramaturgique — son effet passivant la rend, à mon avis, extrêmement suspecte). Ce genre d'œuvres est présenté dans les formes et avec la technique du MKhAT ${ }^{5}$ qui, idéologiquement, se rattache intégralement à la classe des propriétaires fonciers, tant par les acteurs que par la mise en scène. (Pour ceux qui sont un tant soit peu familiers de la technique du Théâtre d'Art, ne serait-ce qu'au plan de la terminologie de toutes ces "rêveries", "états d'âme» et autres, le fondement idéologique de sa technique et de ses formes est, je pense, tout à fait évident.) 


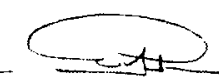

L'effet dépolitisant [de la forme] sur l'auditoire - qui est subreptice, non immédiat, mais résulte d'un long labourage "souterrain" - est aussi évident que la représentation d'un [contenu] soi-disant idéologiquement inconsistant: le fondement idéologique hostile est le même, mais il s'agit des facteurs essentiels que celui-ci détermine et qui produisent un effet sur l'auditoire, à savoir la forme et les procédés techniques.

Sans parler du fait que ce sont eux qui spécifient l'effet produit par le contenu, ce qui est essentiel dans l'art révolutionnaire, et que sans eux le contenu ne pourrait avoir le moindre effet!

Par ailleurs, un genre aussi bourgeois que le cabaret ou le music-hall (visiblement parce qu'ils doivent leur apparition à l'aggravation dialectique de la réaction à l'intérieur même de l'actthéâtre capitaliste, soutenue, comme toujours au cours de l'histoire des révolutions formelles qu’a connues le théâtre, par une intrusion au niveau "académique» des genres «bas» et des formes de spectacles populaires), s'est révélé idéologiquement répondre pleinement, par son principe structurel, à notre propre approche dans la prise de conscience de la création formelle au théâtre.

Sans m'arrêter ici en détail sur la signification de ces principes qui ont marqué la recréation de formes de spectacle cohérent (Le Sage, à cet égard analytique et Moscou, entends-tu? , synthétique mes "montages d'attractions" au Premier Théâtre ouvrier, comme définition des fondements conscients d'un point de vue matérialiste de l'effet produit, ainsi que l'application constructive de ces principes), dont il a été suffisamment question dans la presse, je me référerai ici à leur signification dans la définition du [rôle du cabaret et du music-hall] qui sont, semble-t-il, les seuls parmi toutes les formes théâtrales à avoir le droit de compter sur un avenir quelconque: les «journaux vivants" étant la meilleure arme de la politique [culturelle] des clubs.

Pour revenir maintenant à la question du contrôle idéologique de la forme, il convient de constater deux choses.

L'incompétence totale dans le domaine, il est vrai fort complexe, de la définition formelle des phénomènes du thêâtre et du cinéma en général chez ceux qui en ont la charge. (Je me référerai ne serait-ce qu'aux difficultés colossales qu'il a fallu surmonter pour convaincre le Proletkult d'accepter les principes formels

Rayon et Gnôle [Essai de définition de la carence idéologique dans le domaine de la forme] 
choisis pour La Grève, en raison de son absence totale de repères dans les questions de forme et son incapacité à imaginer qu'une réalisation semblable soit, d'une manière générale, pratiquement pensable et possible, et qu'en plus, elle produise un effet!)

Il est vrai que dans ce domaine d'analyse il n'y a ni méthode ni instructions. La musique, en tant que «forme pure», a davantage de chance à cet égard: devant moi le Glavrepertkom ${ }^{6}$ a un jour écarté une marche funèbre à la mémoire de Lénine pour "procédés de composition relevant du "fox-trot" "!

Mais la faute en revient aux spets, et non aux membres du Parti, et c'est là que réside mon second point. La question de la création de la forme d'une cuvre ne se limite pas pour le créateur à la mise au point, pour lui impérative, d'une solution stratégique permettant d'accomplir la tâche dont il est chargé.

Il est question ici des types de forme et des maîtresproducteurs dont le travail possède véritablement une valeur objective. Dans la langue populaire, il est convenu de les appeler "créateurs". Leur travail comporte, outre les points évoqués, un certain pourcentage d'inconscient, et ce pas seulement pour celui qui s'apprête à effectuer un contrôle - comme dirait un quelconque Perestiani: "Je crée avec mon âme!" Va vérifier cette âme!... Il n'y a pourtant pas grand-chose à tirer au clair son apolitisme (dans le meilleur des cas) est évident d'emblée et, sans même s'intéresser à l'âme, son travail se révèle impossible à corriger, alors qu'il est possible de le faire avec un spets militaire, quand bien même on aurait affaire à un contre-révolutionnaire «dans l'âme».

Il faut bien voir qu'on a affaire à une part d'inconscient, même pour le producteur! Ce n'est pas pour rien que tout le monde "crée" - tel est le résultat de ce manque de prise de conscience de la mécanique de production interne qui aboutit à la création de nos propres valeurs ${ }^{7}$.

Il faut ici faire montre d'une grande clarté objective dans les questions du rôle de l'inconscient en général et de son propre "cas" personnel en particulier.

L'appartenance de classe d'un créateur, qui s'accentue et se manifeste dans sa "superstructure" névrotique individuelle — la "physionomie " du créateur — est le déterminant de son essence 


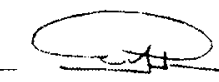

idéologique inévitable qui se dévoile, étant donnée la dictature établie sur le contenu de l'œuvre, dans sa forme. Celle-ci réalise mon idéologie, et non pas celle du commanditaire - elle est pour lui utile, et son effet le dessert.

En analysant la particularité d'une forme et en connaissant les conditions de sa production, on peut remonter à ses facteurs idéologiques, et pas seulement pour démontrer leur «nature suspecte" (bien que dans de très nombreux cas, cela soit également nécessaire, car la nature contre-révolutionnaire d'une forme n'est pas toujours détectable "à l'œil nu»), mais pour en établir les présupposés névrotiques (sortes "d'indices sexuels secondaires", à condition de ranger l'appartenance de classe parmi les indices primaires!), afin de prendre conscience et d'établir une classification détaillée des différentes personnalités parmi les réalisateurs (dont l'appartenance de classe est, en gros, déjà bien connue). Et ceci non dans un but d'extermination ou de représailles, mais, une fois l'essence (les facteurs motivants) d'un producteur parfaitement connus, une fois prises en considération leur inéluctabilité et les possibilités minimales de modifier sa personnalité, sans chercher à le briser, ce qui diminuerait sa capacité productive, dans le but de l'utiliser dans la mesure du possible tel quel (dans des conditions de productivité maximale), en lui assignant une place, un matériau, un domaine de tâches particulières (et quelles spécialités ne sont pas requises aujourd'hui!), en le plaçant dans un contexte où sa "négativité" même deviendrait socialement utile (autrement dit sa satisfaction personnelle coïnciderait avec la satisfaction sociale). Parce que la tactique du Parti dans tous les domaines consiste non pas à éliminer sans restriction tous les "éléments nuisibles», mais à forcer ces mêmes éléments à servir la révolution'. Il en a été ainsi lors de la géniale prise en compte de la psychologie des koulaks et des petits propriétaires fonciers dans la création et l'activité de la Cavalerie rouge qui fut une des armées les plus révolutionnaires, tout en étant composée majoritairement de koulaks ${ }^{\text {II }}$ !

Il doit en être ainsi sur le front de l'art révolutionnaire. Dans la suite, nous tenterons d'introduire une relative clarté dans le domaine de la mécanique "créative» (en recourant aux présupposés généraux des docteurs Freud, Otto Rank et Sachs, sans 
transgresser les limites du "confort" étroitement pratique de leurs théories pour l'analyse proposée) et d'analyser le cas concret de deux films récemment sortis, selon l'orientation choisie ${ }^{\text {III. }}$.

La soif d'agir était chez cet homme (Napoléon) étonnante. Elle avait quelque chose de maladif. Elle était sans doute provoquée par un défaut de son organisme...

... Er comme chez Jean-Jacques, son ambition, son génie étaient engendrés par cette impuissance.

... Si le fils de Laetitia Ramolino a subjugué le monde et versé des flots de sang, cela s'explique par son impuissance.

J. J. Brousson, Anatole France en robe de chambre, chapitre "Limpuissance de César" ".

On peut grosso modo diviser la production artistique en deux catégories: le produit artisanal et ce qu'on appelle le produit «de la création" - suivant la façon dont on évalue leurs producteurs.

Le cinéma étant de tous les arts le plus proche de l'industrie, c'est lui qui départage le plus nettement ces deux espèces.

Celles-ci ne se caractérisent nullement par leur qualité, puisqu'elles sont au fond sans commune mesure, mais par leur différence génétique, indépendante de la volonté de leurs créateurs.

Les premières peuvent plaire ou non, être techniquement brillantes, avoir du succès; les secondes également, mais elles possèdent en plus ce quelque chose qui saisit le spectateur à la gorge, le touche, produit sur lui une impression "profonde».

Les premières sont le produit d'une évaluation cérébrale $\mathrm{du}$ matériau principal que constitue le spectateur de cinéma, le consommateur, et [procèdent] d'une utilisation habile des matériaux permettant de produire un effet - c'est un métier comme un autre, le choix délibéré d'une forme d'artisanat.

Les secondes sont pour leur producteur une nécessité physique et psychologique, car elles sont un acte de sublimation pour les névrosés que sont immanquablement les maîtres qui, tout en partageant avec les premiers les mêmes procédés stratégiques, ont ce quelque chose de "particulier» qui les en différencie nettement. 
La base du premier groupe est constituée d'hommes sains, modérément lirnités, dont les meilleurs représentants pétillent d'une joie animale d'affirmation de la vie, jouissent d'une musculature de sportifs légèrement bornés ou, dans le cas des plus médiocres, boborykent ${ }^{10}$ par tradition leur production mensuelle (la productivité, par opposition à la rareté, est tout à fait caractéristique de cette catégorie, quel que soit le niveau de maîtrise formelle).

Dans le second cas, nous avons affaire à quelqu'un de "malsain ". Bien que les travaux de Bleiler et Kretchmer ${ }^{11}$ aient une fois pour toutes effacé la frontière entre individu "malade" et individu "sain», en remplaçant la distinction qualitative entre normal et anormal par une distinction quantitative du degré de développement et par la prédominance de certains composants du psychisme général (déterminé par sa structure organique), la désignation a malgré tout perduré par convention et continue de signaler le moindre écart par rapport à la norme orthodoxe.

Nous avons donc affaire à un névrosé dont la névrose («la tare») est une sorte de catalyseur des capacités créatrices, renforcées par la puissance de l'énergie de classe qui se révèle, par ces voies sinueuses, à travers sa superstructure ultime de classe une névrose individuellement marquée. La libération de cette réserve d'énergie, qui s'autodévore lorsqu'elle reste à l'état de potentialité et qui, sinon, s'effectue en surmontant par la volonté le penchant à satisfaire de façon primitive et directe les tendances névrotiques, définit l'acte de sublimation en tant que mécanique permettant de transposer la réalisation d'une attirance anormale ${ }^{\mathrm{IV}}$ (qui se manifeste quand même* sous la forme de perversions qui sont le résultat de l'intrusion dans l'acte de création d'éléments conscients mais inacceptables, refoulés dans le domaine de l'inconscient) de la sphère de l'autosatisfaction vers celle de la satisfaction sociale, en recourant aux facteurs de la vie sociale qui, tout en partant du même présupposé génétique de recherche de la satisfaction (ils peuvent même se réaliser sous une forme identique!), sont déjà socialement concevables de par leur utilité.

* Mots, groupe de mots ou expression apparaissant en allemand, anglais, français ou latin dans le texte original russe.

Rayon et Gnôle [Essai de définition de la carence idéologique dans le domaine de la forme] 
Un sadique devenu un éminent chirurgien — voici l'un des meilleurs exemples de sublimation.

Un tel acte non seulement supprime la névrose, du fait qu'il brise le "cercle magique" de l'asocialité - condition indispensable du développement et indice le plus caractéristique de la névrose —, mais, en renforçant la volonté et en libérant l'énergie qu'elle contient, élargit sensiblement l'intensité de sa capacité d'action.

En ce qui concerne la production de l'un et l'autre camps à proprement parler, il découle de ce qui vient d'être dit que non seulement le premier ne fournit pas un art sain et l'autre malsain, mais que, d'une manière générale, tous les avantages sont du côté du second car, en plus de la salubrité parfaite du produit de cette sublimation, nous obtenons, du fait du processus de transfert de l'individuel vers le social (cf. Freud), des manifestations qui, outre leur salubrité, sont forcément socialement utiles, quand bien même ne seraient-elles pas toujours obligatoirement d'une grande portée sociale.

L'un des domaines où les possibilités de sublimation sont les plus vastes, tant par l'intensité psychologique que par la portée sociale, est celui de l'art. Et la mise en scène y occupe sans doute la première place, car elle se fonde en premier lieu sur [ce processus de sublimation] et offre les plus larges possibilités de concrétiser presque totalement les facteurs motivants cachés, tout en leur accordant une finalité complètement différente et en les " neutralisant " par leur utilisation sociale.

Par ailleurs, le dépassement qui accompagne la sublimation développe un trop plein d'énergie qui augmente considérablement les manifestations de volonté et d'organisation - qualité principale d'un réalisateur - en lui communiquant de surcroît cette passion et cette avidité particulière à capter la vie, l'auditoire, etc. qui sont la marque des réalisations majeures.

Il ne convient pas de chercher des fondements "mystiques" à la "profondeur" particulière de ce genre d'œuvres — les choses sont beaucoup plus simples: en étant obligé d'inclure, ou plus précisément, en se basant sur le matériau que lui propose son inconscient (pour les trois quarts de façon certainement intuitive, autrement dit en se laissant guider soi-disant inconsciemment 


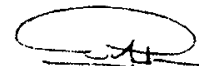

par ses "goûts et penchants" dans le choix et l'élaboration de ce matériau, du coup déterminé précisément par ceux-ci), le créateur établit un lien avec son auditoire non seulement en travaillant émotionnellement sa conscience, mais en attirant directement dans le «jeu" l'inconscient du spectateur qui lui fait écho dans la mesure où chacun possède sa part de matériau névrotique sublimé, à un degré simplement différent.

La représentation du produit de cette sublimation propre au récepteur suscite chez ce dernier cette sensation de "plaisir" que l'on éprouve à la réception de ce qu'on appelle les "grandes cuvres".

Le fondement de ce plaisir tient, ici comme partout ailleurs, à la présence d'un élément d'économie (la satisfaction modérée n'est pas encore le plaisir - celui-ci n'intervient que lorsqu'il y a relatif excès : la ménagère qui a eu juste assez pour tout acheter, est calme, tandis que celle qui est parvenu à économiser un sou est heureuse). L'économie est profit. Dans ce cas particulier, le profit économique est assez proche, et par bonheur plus primitivement compréhensible et plus immédiatement perceptible que dans le cas du mot d'esprit (Witz) — à l'analyse duquel Freud a dû consacrer un livre entier. Le spectateur se voit proposer une résolution toute prête, sublimante, de ses propres désirs qui cherchent une issue, qu'il peut trouver en "participant en communion" à ce qui lui est présenté tout en faisant l'économie de l'imagination et de tout le travail préalable nécessaires.

Et ceci avec un tel degré d'intensité que la satisfaction de son activité illusoire de spectateur, indépendamment du fait qu'il y ait été entrainé par des voies illusoires (front de droite) ou non (front de gauche) (car tel est le but et le fondement de tous les types de spectacles qui produisent un effet, à la différence des spectacles d'action, qui toujours entraînent réellement le spectateurcoparticipant) ne le cède en rien par son intensité à ce qu'aurait produit la manifestation réelle d'une activité équivalente. C'est cette fiction qui détermine la nocivité sociale de tous les types de spectacles et simultanément conditionne également leur vitalité. On voit clairement maintenant où se situent les fondements de la "profondeur" de l'effet produit (dans la mesure où il s'agit de 
sub-conscient, le recours à ce terme "métropolitain" n'est pas un hasard!) et les fondements de cette impression particulière dont il était question plus haut.

Le réalisateur américain moyen produisant en quantité incalculable des productions plus ou moins grosses peut servir d'exemple de notre premier type.

Cecil B. deMille et Douglas Fairbanks sont, à mon avis, les parangons de ce style. Le premier, "Protazanov idéal», comme quelqu'un l'a dit, est le nec plus ultra de la ligne "Boborykine" qui comprend toute la pléiade de nos réalisateurs professionnels d'autrefois (Gardine, Sabinski, Ivanovski, etc.)

Douglas est bien sûr l'idéal de la ligne "musculaire" et ceci non pour la musculature du Voleur de Bagdad, mais pour le caractère "musclé" de l'évaluation de ses propres œuvres.

C'est tout simplement la salubrité physique des présupposés de son travail qui séduit, un point c'est tout ${ }^{12}$.

Parmi nos réalisateurs, Leo Mur a sans doute quelques chances dans cette voie, à condition qu'il parvienne à maîtriser la forme cinématographique et qu'il comprenne que les réalisateurs du genre «bastkeballeurs" n'ont pas à s'attaquer à un matériau réservé aux névrosés ${ }^{13}$.

Dans le domaine de la production, ce groupe est, en Amérique, certainement le plus frable d'un point de vue de classe et chez nous celui qui exécute le plus "honnêtement»... les instructions du Glavrepertkom. Ici comme là-bas, ce n'est qu'une question d'ancienneté.

Mais ce ne sont pas des "combattants" indépendants, ni des "personnalités" combatives.

(Doug, ce "brave gars" international, n'aura jamais les prétentions d'un Chaplin.)

Le second groupe est constitué de créateurs d'une autre "trempe», d'une autre passion, d'un autre pathos. Ce ne sont plus des experts-comptables en psychologie salonarde, à l'horizon de boxeur, quand bien même seraient-ils extrêmement qualifiés.

Griffith et Eric von Stroheim sont ici, je crois, en tête.

La puissance et la portée de leurs œuvres s'effectuent au détriment de défauts énormes qui altèrent leur fondement nerveux. 
L'aspect formel de leurs œuvres se caractérise par une forme manifeste de sadisme sublimé, ce qui les rend incomparables par l'effet bouleversant qu'elles produisent.

La cruauté et l'implacabilité - le sadisme est la principale névrose indispensable à un réalisateur en général: sous le rapport des produits sublimés du sadisme, le réalisateur est comparable au chirurgien et... au boucher.

(En raison des exigences spécifiques de la profession et de la production, la "vivisection" du matériau et la "violence» sur le spectateur sont [indispensables].)

Mais dans le cas de Griffith et de Stroheim, elle marque également de son empreinte "l'individualisation" de ces créateurs, ici même, à travers le choix du matériau et les procédés de son traitement, pour aboutir à un chef-d'œuvre de sublimation.

Et ce n'est seulement que dans un dépassement par sublimation que des chefs-d'œuvre d'une telle passion et, à leur manière, d'une telle portée sociale, que Naissance d'une nation ou Dans la fournaise de la guerre ${ }^{14}$ ont pu être réalisés. Des preuves? Lisez les descriptions de Naissance d'une nation où Griffith est plus féroce que des marchands d'esclaves, regardez une fois encore ce «document humain» évident qu'est Le Lys brise' ${ }^{15}$, production non sublimée entièrement et qui, pour cela, provoque le dégoût (il sera question plus loin de choses semblables), tout en dévoilant les pulsions habituellement masquées de son créateur - et qui produit une impression presque aussi désagréable et effrayante que la contemplation du sadisme vulgaire de Bassalygo (la scène du fouet dans De l'étincelle naquit la flamme et celle de torture dans L'Arrière-front rouge ${ }^{16}$ !).

C'est en particulier dans Intolérance (titre déjà caractéristique), América, enfin Les Deux Orphelines ${ }^{17}$ et Loin à l'est ${ }^{18}$ que l'on voit à quel point, en dehors de ce que l'intrigue met en relief (à propos, en énumérant uniquement les situations traitées, on pourrait voir qu'elles tournent presque toutes autour des thèmes de l'intolérance et de la violence!), Griffith est impressionnant et extrêrnement cruel dans les détails: les genoux ouverts de Lilian Gish après l'accouchement quand son bébé est mort, ou bien le procès dans Les Deux Orphelines, lorsque Lilian demande que la sentence de mort soit prononcée à voix basse afin 
que sa sœur aveugle, assise dans la salle au milieu d'une foule immense, n'entende pas, sont inoubliables justement à cause de la cruauté du réalisateur. (Cette dernière scène, bien plus forte que celle de la guillotine, fut supprimée chez nous pour des raisons de "censure ".) Le fait d'être capable de tels détails de mise en forme se paie nécessairement par un dérèglement de la constitution nerveuse normale du cours de la vie. Ce n'est pas un hasard si Griffith travaille 18 heures par jour, et que cela fait 15 ans qu'il ne trouve pas le temps d'avoir des "aventures"!

Eric von Stroheim, qui dépasse à mon avis Griffith par la cohérence supérieure de sa personnalité, ne suscitera probablement pas de doutes. Ceux qui savent ce qu'il joue et comment il le joue et qui ont vu ce qu'il réalise et comment il le réalise, ne discuteront pas. Prenons par exemple dans Adjudant de FranzJosef $f^{9}$ les scènes comme la tentative de viol de la jeune fille dans le manège endormi ; le patron du manège qui; à l'aide d'un couteau, interrompt à mi-parcours le mouvement de la main de sa femme en direction du pain ; les minuscules morceaux de saucisson, filmés en détail, qu'il lui laisse à côté de sa bière et de l'énorme sauciflard qu'il dévore; la mort de la femme du clown; l'histoire du bossu - partout on trouve un élément actif de cruauté dans le traitement (comparer au traitement totalement opposé de ces mêmes moments chez le réalisateur d'Humoresque dont il est question plus bas) qui ne le cède en rien aux scènes d'amour lyriques les plus captivantes: quel travail de scalpel subtil dans leur "crucifixion" psychologique!

Mais si le sadisme est indispensable au réalisateur en tant que trait professionnel, cela ne signifie nullement qu'il définisse une fois pour toute sa personnalité névrotique. Chaplin et Frank Borzage ne sont pas des exemples moins caractéristiques de ce même groupe, bien qu'ils soient totalement opposés aux deux premiers.

À propos, personne chez nous ne connaît Borzage, bien que son meilleur film, Humoresque, primé en Amérique, soit sorti chez nous cette saison (sous le titre $A u$ nom de quoi ?), criminellement privé de publicité, non remarqué et non reconnu par personne, bien qu'il constitue un exemple rare de subtilité et de maîtrise, principalement dans le traitement et le travail de réalisation $^{20}$. 
On fait une publicité effrénée pour dieu sait quelles Nelly et autres Monique Lherbier ${ }^{21}$, alors que des œuvres réellement de premier plan, comme par exemple celles de Stroheim, passent totalement inaperçues (notamment Foolish Wives qui est le meilleur film étranger sorti ces deux dernières années chez nous, si l'on ne tient pas compte de Fête des cow-boys en Pensylvanie et de Nanouk $^{22}$ ).

Chaplin et Borzage, par leurs thèmes, par leur doux lyrisme, la féminité de leur douleur passive, font pencher en faveur de stimuli névrotiques directement opposés aux premiers, du côté des souffrances rêveuses de Rousseau et de Beardsley.

La pauvresse boiteuse, le violoniste au bras arraché pendant la guerre, Max le gentil fou, les malheurs et les persécutions de la famille juive, son rôle passif dans toutes les épreuves, le Muttercomplex* terriblement souligné (justement par la mise en scène), etc. chez Borzage (malheureusement, je ne connais qu'un seul de ses films).

Quant à Chaplin? Ce n'est pas un hasard si son masque se fonde sur un objet passif de cruauté - l'immigré italien qui est, en Amérique, l'objet de raillerie nationaliste et sociale: nous ne ressentons presque pas cet aspect-là du masque de Chaplin, celui de l'humilié, en tout cas nous n'en avons pas suffisamment conscience: pour nous il est comique sans objet (voir à ce sujet un des articles consacrés à Charlot dans Le Crapouillot, Paris, 1921).

Pourtant, comme l'écrit Willy Haas («Einwande gegen Chaplin ", Der Kinematograph, 1923, n 848), "son trébuchement est à la fois le plus absurde, le plus maladroit, et le plus méchamment calculé (boshaftgelenkigste*) du monde, il arrive souvent que dans un même film, il fasse appel simultanément à notre compassion et à notre jubilation cruelle, à notre humanité et à notre inhumanité". Et rien sur le rire! C'est parce que l'œuvre de Chaplin, qui touche au thème des "humiliés et des offensés" avec une force qui ne le cède en rien à Dostoïevski, donne des raisons de soupçonner chez lui les mêmes maux que ceux qu'a dévoilés Le Souterrain d'un génie à propos de Dostö̈evski ${ }^{23}$. Quoi qu'il en soit, les passages les plus remarquables chez Chaplin son lyrisme - valent les passages les plus "touchants" de 
l'auteur des Pauvres gens. En outre ce n'est pas un hasard si Chaplin, qui joue toujours des rôles de serviteurs, est toujours battu, et quand c'est lui qui frappe, c'est plutôt pour démontrer qu'en fin de compte, c'est toujours lui, socialement, qui est battu. Comparez Chaplin avec Monty Banks dans un film récemment sorti chez nous, Automobile $n^{\circ} 13^{24}$, où il apparaît sous le même masque de l'Italien et dans des situations similaires (bagarre, passage à tabac, grandeur d'âme, etc.) et où, à tous égards, il fait figure (en tant qu'acteur) de "parfait charcutier" dans sa médiocrité petite-bourgeoise. Je ne sais si le rappel de la liaison, qui dura deux ans, de Chaplin avec Pola Negri durant son séjour à Hollywood, rendra cette réflexion plus convaincante. Le choix même d'une "femme-vampire" professionnelle de ce type est, à lui seul déjà, significatif de certaines "options sexuelles». En passant aux représentants de cette catégorie de réalisateurs de notre cinématographie qui demandent non un barème mais une analyse, je dois préciser qu'il n'est pas tant question de l'importance de leurs travaux que de la présence d'un matériau symptomatique permettant de tirer des conclusions et de procéder à une analyse, certes sur un ton peut-être trop élevé en regard de la signification absolue des travaux analysés. (D'autant plus que ce groupe comptabilise pour le moment le plus grand taux d'échecs.)

Il découle de la partie précédente que les œuvres significatives par leur valeur sociale n'ont pu être réalisées par leurs auteurs qu'au prix d'une certaine défaillance de leur équilibre névrotique ("le prix du feu de Prométhée», comme le dit Gontcharov dans des cas semblables). En dehors du cinéma, les travaux de recherches dans ce domaine sont légion - je suis semble-t-il le premier à appliquer ces méthodes aux réalisateurs de films, en tout cas certainement dans l'optique indiquée au début.

Mais... il ne s'ensuit pas pour autant qu'il suffise d'être névrosé pour devenir, et même pour continuer d'être réalisateur.

La présence de ce facteur de départ ne garantit pas encore contre l'" échec ".

L'échec d'une œuvre, son infirmation sociale ou, plus précisément, son invalidation peut se produire, en dehors des conditions techniques et des limites imposées par le sujet et la thématique — non examinées ici — dans deux cas repérés par moi à 


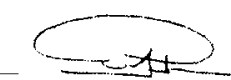

ce jour: lorsque l'œuvre n'atteint pas le stade achevé de sublimation et, cas plus complexe, lorsque la névrose personnelle, de par sa spécificité, non seulement ne se combine pas avec les principales qualités professionnellement utiles (aspect technique), mais, même sublimée, n'est pas ajustable ou n'est pas ajustée avec ce que requiert la socialité.

Puisque l'individualisme dans la création de la forme comme concrétisation de ses pulsions, responsable de ses convoitises - , en se socialisant dans le processus de sublimation, peut toutefois (tant au niveau de la définition formelle - de la structure formelle - processus purement conscient mais dont le facteur déterminant s'enracine dans le subconscient, que dans le résultat final) ne pas coïncider avec les orientations sociales obligatoires.

N'étant pas déterminée à toutes les phases du processus productif de la "création" par une orientation idéologique globale, mais par une orientation personnelle, individuelle (a priori toujours suspecte), l'ajustement ultérieur de cette production avec la socialité en question est fort aléatoire et exige non seulement une grande "dextérité", mais en tout cas un contenu si avantageux qu'il soit possible de s'insérer dans le "socialement utile", en se faisant ainsi « remorquer".

Malheureusement, ce procédé est presque "général» à toute notre cinématographie.

Les deux types mentionnés s'appliquent précisément à deux cas d'échec formel qui concernent la personnalité du réalisateur (et non l'aspect technique formel, sous le rapport duquel l'un des deux est même exemplaire).

Ce sont les fondements de ces échecs, sous l'aspect ici envisagé et du point de vue indiqué, que j'essaierai d'analyser.

"Un malheur ne vient jamais seul, il amène toujours son cousin avec lui " - ainsi s'exprime apparemment un Français dans une comédie de Lessing.

Dans le cas des névroses, on a toujours à faire, comme ici, à la présence de plusieurs complexes enchevêtrés.

À côté de la névrose primaire, fondamentale, professionnellement déterminante, névrose du «réalisateur ", comme je l'ai déjà dit, on en trouve toujours une seconde, plus forte que les autres, 
qui donne au réalisateur son empreinte individuelle - sa physionomie.

Dans deux cas, il s'agit du sadisme, dans deux autres, du masochisme.

Ces deux groupes sont à mon avis (du moins selon ma vision de l'art comme forme de violence), probablement les plus productifs, non seulement d'après les données statistiques, qui d'ailleurs sont totalement inexistantes, mais en théorie aussi, en raison de leur rôle particulièrement stimulant. [Il en va ainsi] tant pour celui qui produit l'effet, dont le rôle est évident dans le premier cas, et qui dans le deuxième est une réaction naturelle transformant la passivité masochiste en sadisme actif ("tant qu'à agir, autant agir à fond»), ce qui, sous l'aspect de la production, revient au premier cas. Que pour le récepteur qui, dans le premier cas, est manifestement activé au maximum (à condition bien sûr d'une orientation ad hoc) et qui lui-même s'active au maximum grâce aux principes actifs des instincts qui sont suscités en lui et qui s'opposent à ce qui lui est montré ${ }^{v}$.

Le fétichisme ("Dès le lever du jour, vous êtes déjà sur pied, et je suis à vos pieds ${ }^{25}$ ) constitue le terreau sexuel des prémisses d'une vision du monde idéaliste — d'une divinisation désœuvrée de l'idée - voilà, je crois, une des névroses fatales pour un réalisateur à notre époque d'ardeur matérialiste. Vestige de notre passé esthétisant. L'idéalisme passif de l'image "à vos pieds" est directement opposé à l'activité tractoriale du labourage, en tant que modèle pour nos réalisateurs révolutionnaires.

Je ne sais pas s'il existe déjà un portrait psychanalytique de Tchatski (comme cela a été fait pour Hamlet, Don Carlos, Edipe - l'Inzest-problem* jouit dans cette direction d'une fortune particulière), mais je crois que dans le cadre d'un travail de ce type, le vers cité plus haut du Malheur d'avoir de l'esprit ne passerait pas inaperçu. Et n'oublions pas la physionomie de la jeunesse d'autrefois, baignée de l'idéalisme du jeune romantisme allemand à la Novalis, et encore davantage la configuration sociale du fétichisme propre au groupe dont Tchatski, inspiré par les décembristes, était le représentant. Nous connaissons l'appréciation que les marxistes portent sur la valeur sociale de cette flamme fanatique qui flamboie sans brûler, de ces fétichistes de 
l'idée, comme de la plupart des "conjurés». Nous ne suivons pas la même voie que les décembristes.

Et si l'idéalisme ne sera jamais admis comme sujet au cinéma, grâce aux ciseaux du Glavrepertkom, son unique refuge deviendra la forme, encore non astreinte idéologiquement à la censure en raison de l'incapacité dans laquelle se trouvent ceux dont le devoir est de juger de ses facteurs stimulants structurels - car dans le domaine de l'évaluation formelle des ouvres, le même Tchatski dit bien: "Qui sont ces juges?" Mais alors, il se trahit complètement, car l'idéalisme dans la forme est stylisation voir l'art religieux propre à l'idéalisme de toutes les périodes authentiquement fétichistes (Byzance, le Moyen-âge, le Trecento, les dieux nègres, etc.).

Dans son refus de reconstruire intentionnellement le réel au bénéfice de l'irréalité fictive, au lieu de se colleter de façon matérialiste à la réalité actuelle, la stylisation est directement opposée à l'utilitarisme. Chez le névrosé "fini ", le rapport à la réalité se révèle dans ses rapports particuliers avec le monde extérieur.

Il est significatif que le premier élément de matérialisme décelé dans mon travail théâtral m'ait amené à cette première conclusion (qui fut relevée par $S$. Trétiakov) que «le théâtre des attractions n'est pas un théâtre d'un style achevé, mais un théâtre d'action de classe efficace, aux buts utiles et pleinement conscients » (Oktiabr' mysli [Octobre de la pensée], $\mathrm{n}^{\circ} 1$, janvier $1924^{26}$ ).

En général, le concept de "style " appliqué aux formes de l'art révolutionnaire est très problématique - le style est le produit d'une méthode canonisée, constituée et finie (dogmatique) —, la forme de l'art révolutionnaire répond au caractère changeant de l'essence de la tactique (dynamique).

En apportant à chaque instant des solutions aux problèmes actuels, il ressemble au dynamisme des formes de notre État et à celui de sa constitution politique intérieure.

La Révolution a remis l'art en question de façon beaucoup plus profonde, à mon avis, que dans le simple but de créer un style propre à l'URSS, qui peut intégrer Werman ou Lubke à égalité avec la Renaissance, Louis XV - seizième du nom ${ }^{27}$, et autres. Il s'agit de réexaminer radicalement son sens, sa destination et sa "situation" sociale — d'abolir la vision de l'art 
comme superstructure $\left[{ }^{28}\right]$, essentiellement comme couche réfléchissante - pour, à l'avenir, le "rabaisser" au rang de manifestation subalterne spécialisée de la tactique de combat, ainsi qu’à d'autres fonctions socialement utiles (en premier lieu pédagogiques et cliniques): [il sera] sous l'aspect de sa production consciemment construit, loin des illusions liées à la "création", afin d'effectuer grâce à sa puissance énergétique des commandes sociales, comprises de façon artisanale ${ }^{\mathrm{vi}}$.

C'est autre chose que le style!

L'art stylisé (comme limite de la manifestation d'un style dans la révélation de la méthode inaltérable d'une divinité) [est] autoreligion.

La stylisation de formes toutes prêtes vole plus bas - c'est déjà du fétichisme primitif.

Stylisé par sa forme - produit de sublimation du fétichisme de son réalisateur qui le définit, dans le domaine de l'approche formelle, comme idéaliste - tel est Le Rayon de la mort, rejeté par notre socialité, et probablement inacceptable socialement en raison du fétichisme excessif de sa forme.

Je préfere attirer uniquement l'attention sur l'adoration rituelle de l'américanisme ${ }^{29}$ — produit de sublimation purifié, mais je ne peux pas ne pas relever le matériau beaucoup plus "périlleux» qui relève du fétichisme primitif: la prééminence du pied et de la chaussure pointue, sous la bannière duquel on peut ranger tant Le Rayon que West ${ }^{30}$.

Sans parler des jambes inoubliables de Khokhlova, "vis motrix*" de ces œuvres, il convient de relever l'attention que porte Kouléchov (sans s'arrêter au "culte" insistant de la bonne chaussure dans son atelier) - tant dans la mise en scène que dans son système de formation cinématographique - aux déformations spatiales des jambes du modèle qui précisément ne visent pas la marche, laquelle détermine le déplacement, ni les muscles des jambes comme principaux ressorts moteurs des manifestations motrices du corps en général, mais qui affectent un plan "esthétique», autrement dit ont une visée non utilitaire (dans ce cas moteur), due à d'autres facteurs déterminants.

La prise en compte consciente, et non la perception contemplative, de la jambe dans sa fonctionnalité (son rôle décisif dans 


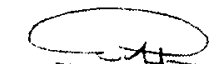

la globalité du mouvement), étant donnée l'attention particulière dont elle fait ici l'objet, ne pouvait pas ne pas dévoiler le secret de la mécanique du mouvement en général auquel elle sert de clef. Mais c'est justement l'approche contemplative à laquelle nous avons ici affaire qui détermine tout le système du mouvement chez Kouléchov.

Le système du mouvement chez Kouléchov, c'est de l'idéalisme dans le domaine de la technique du mouvement.

Sans analyser en détail le système de travail du modèle selon Kouléchov, auquel je pense consacrer, en même temps qu'à la critique d'autres systèmes, un essai particulier intitulé Principes d'organisation d'ans le travail au cinéma (où j'exposerai les bases matérialistes de mon système de mouvement au plan de mon usage personnel, que j'ai ultérieurement complètement rejeté en raison de l'approche totalement différente dans ses principes que j'ai développée pour le cinéma, ainsi que la question des studios de cinéma et celle des collectifs ${ }^{31}$ ), je me bornerai à indiquer qu'elle [la technique du mouvement chez Kouléchov] ne se fonde pas (probablement en raison de ce qui a été exposé plus haut) sur l'organisation scientifique du processus de travail du modèle, qui est le seule pensable d'un point de vue matérialiste en tant que travail visant à dépasser les résistances physiques (les moments de gravitation et d'inertie du corps) par des manifestations volontairement affirmées.

Les prémisses [de ceci] s'appuient sur de longues années de pratique, et ont été découvertes par des Allemands consciemment orientés vers le spiritualisme - cf. les travaux de Rudolf Bode et Ludwig Klages - qui, il est vrai, tentent d'introduire subrepticement leur métaphysique nationale dans le domaine des fondements théoriques.

Au bout du compte, on a manifestement affaire non seulement à une extraordinaire stylisation, mais, dans la caractéristique des procédés du mouvement de l'école kouléchovienne, à une méthode de stylisation sous influence étrangère ([prenant pour modèle] des phénomènes tirés non de notre réalité, mais de l'écran américain). Les manifestations de cette méthode sont inévitables en raison de la rupture de celle-ci avec sa base principale - le déterminant matériel et technique des fonctions 
motrices, et sont déterminées par un goût qui, de surcroît, a non seulement une autre visée sociale, mais une autre nationalité!

Peut-être Kouléchov a-t-il été séduit par la méthode descriptive de Darwin (livre de chevet de tout acteur américain, à commencer par le petit Wesley Berry que nous connaissons par Juddy trouvé (cf. Klumph, Screenacting, 1922 ${ }^{32}$ ) — il ne faut pas oublier que De l'expression de l'émotion chez l'homme et l'animal ne va pas plus loin que la fixation d'indices extérieurs de l'expression et s'efforce d'expliquer les causes de leur apparition sans analyser aucunement leurs rapports dynamiques ${ }^{33}$.

En décrivant les fonctions de certains muscles, et en utilisant pour cela les travaux de Duchenne, Darwin ignore absolument le principe dynamique fondamental, formulé en 1852, qui en découle comme observation: "L'action musculaire isolée n'est pas dans la nature* ", à l'établissement duquel ont servi finalement toutes les expériences d'électrisation des muscles utilisées par Darwin.

Il est significatif que Kouléchov, en stylisant de manière idéale l'Amérique et sa production, de surcroît pas celle de "première catégorie" (selon notre division), c'est-à-dire finalement une Amérique très superficielle, et apparemment aisément imitable (imiter un film policier commercial n'est pas du tout la même chose qu'imiter, par exemple, Stroheim!), ne peut en rien reproduire matériellement (factuellement) ce qu'elle a de plus séduisant: son essence captivante (que ce soit dans le genre policier ou comique).

Bien qu'il ait eu deux fois entre ses mains les possibilités les plus absolues d'atteindre un résultat formel, tant par ses propres ajouts de situation au scénario, que par le métrage et l'idéologie ("sans surveillance"), les trois quarts du Rayon de la mort et de Mister West ont été dévolus à un matériau idéologiquement vide! Les "passages réussis", comme la course-poursuite dans West et le faubourg dans Le Rayon, ne sont que des exemples de maîtrise technique qui soulignent d'autant plus l'absence de tempérament fondamentale du réalisateur, par comparaison avec le reste.

Mais si le style, plus précisément la stylisation, peut-être touché par le fétichisme, porteur de l'idéologie de la passivité, ce n'est pas le cas de l'action, et encore moins de l'effet produit. 
Ce qui est remarquable ici, c'est la façon dont un tel sujet porteur de présupposés passivants penche, de manière presque maladive, presque comique dans le choix du matériau, vers ce qui lui est organiquement opposé - vers le degré superlatif de l'activité.

En plus de l'épithète permanente des écrits et des déclarations de Kouléchov -_ "énergique» - , regardez cette accumulation de situations d'activité maximale, allant même à l'encontre de tout sens commun : toutes ces courses-poursuites, bagarres, fusillades, rixes, etc. Plus elles sont actives dans leur finalité, plus elles témoignent par leur stérilité formelle de l'impuissance sur laquelle elles se fondent.

Cette incapacité à produire un effet formel apparente Kouléchov à un autre fétichiste, "antagoniste" par l'école à laquelle il appartient - Vertov, fanatique de la machine en général, et de la caméra en particulier, dont toutes les œuvres, formellement impuissantes, sont marquées par l'absence de passion et l'asexualité (passez-moi le mot*).

Ce n'est pas de là que peut sortir l'art cinématographique de l'effet maximum dont nous avons besoin et il est peu probable qu'il faille en attendre un "ciné-poing". L'échec de ce qu'il y a de plus essentiel, est manifeste, malgré une "réussite technique" brillante (à proprement parler, qu'est-ce que cette expression, dite et redite, signifie, et où est au fond son sens, si la technique - en dehors de la fabrication, réellement irréprochable - doit aussi dorénavant et sera évaluée en premier lieu du point de vue de "l'idéologie" ?)

Lespoir repose entièrement sur la collaboration avec un étatmajor de collaborateurs d'une base idéologique différente "une autre névrose de classe" - et sur la spécificité des tâches stratégiques qui lui seront confiées pour mener à bien des opérations tenant compte de la transposition de l'élément fétichiste sur des objets appropriés.

Ce qui deviendra certainement socialement utile, comme le sont sans doute dans certaines limites les dithyrambes contemplatifs des Kino-pravda de Vertov (dans leur caractère particulier - fétichiste en raison de la tâche elle-même). 
Si, dans le cas analysé, nous avons avons affaire à un échec de la production, comme conséquence d'une névrose incompatible avec les conditions de notre "aujourd'hui stratégique" (comme s'exprime Vertov), fétichisme qui a conditionné : 1) l'idéalisme personnel dans l'approche idéologique tant de la technique que de la forme ; 2) la passivité là où il fallait davantage d'activation (bien que par un autre aspect, ce soit surtout une fête de la photogénie, ce qu'est au fond Le Rayon de la mort - fête certes de l'Annonciation plutôt que du Premier Mai!), dans l'autre cas, nous avons affaire à un échec comme résultat de causes purement internes dans le "déroulement" du processus créatif. Une autre névrose est à sa base. Ce qui est curieux ici, c'est son rôle dans la photogénie, cette texture, dirais-je, de la ciné-forme ${ }^{34}$.

La Course à la gnôle. Les prétentions sont des plus modestes - celles d'un film d'agitation sous la forme d'une comédie de mours.

Ce qui a été trouvé à tâtons, de façon largement inconsciente, c'est une formule primitive mais correcte pour résoudre les tâches de propagande superficielles: tout en agissant sur les réflexes inconditionnels, offrir parallèlement, les combiner avec des phénomènes, pour lesquels il serait souhaitable à l'avenir d'établir un réflexe associé approprié.

La pratique de la technique du spectacle d'agitation est une technique visant à susciter des réflexes conditionnés - tout à fait comme chez Pavlov.

Malheureusement, aucun effort n'est fait pour prendre conscience en détail de ce procédé d'agitation lapidaire, utilisé en fin de compte plus ou moins consciemment: il est même au contraire partout appliqué de façon en quelque sorte inconsciente - aurait-on craint d'arracher à l'art sacré son voile aérien de création et d'inspiration, devenu aujourd'hui linceul? Allez savoir! Au bout du compte, ou bien ce procédé traîne à la remorque (sans rapport avec Pletniev ${ }^{35}$ ) de matériaux encombrants et insignifiants dont plus personne n'a besoin, dans des cinémollusques au métrage interminable (Palais et Forteresse ${ }^{36}$ ), ou bien les procédés d'application de ce principe dénotent une grossièreté extraordinaire qui "choque" le spectateur socialement le mieux disposé (cf. à ce sujet [ce qu'en disent] les 


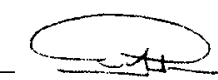

rabkors $\left.{ }^{37}\right)$ : voir le sport bassalugesque au milieu des braises de l'ancienne flamme de l'ex-Proletkino.

Alors qu'en étant suffisamment conscient de ce principe et en orientant l'énergie et l'inventivité dans la bonne direction (celle de sa mise en valeur), on peut parvenir à des procédés très raffinés et sophistiqués qui ne relèvent plus de l'agitation grossière, mais de la propagande subtile (telle est justement la demande aujourd'hui).

En quoi consiste donc l'échec de La Course? En ce que la voie saine, esquissée intuitivement pour réaliser la tâche d'agitation, a subi, sans doute en raison précisément de cette intuition, au cours de la mise en forme de cette tendance, une déviation intérieure brutale dans sa visée factuelle (finale), déviation qui de surcroît s'est exercée à travers les même associations que $L e$ Rayon.

Room souffre d'une névrose - je ne sais pas précisément comment la science la désigne - qui lui fait trouver du plaisir dans ce qui est répugnant, principalement en rapport avec le processus de prise ou de rejet de nourriture (nourriture «dégoûtante», vomissements, souillures à l'aide de nourriture, etc.) et avec la laideur sous l'aspect d'une transgression physique des normes, en particulier dans le domaine de la "texture" (de la peau et autres tissus - gourme, difformités, maladies de peau, vieilles hardes en lambeaux, pouilleuses, tissus vestimentaires eux-mêmes lépreux). Lélargissement des limites définit, comme nous le constaterons encore, les deux aspects de la névrose exposés ci-dessus qui caractérise Room.

La sensation de dégoût se fonde sur la sensation de chaos, résultat de l'inorganisé, de ce qui est contraire aux processus se déroulant normalement, de façon organique. Dans le dégoût, l'hostilité apparaît du fait que toute transgression des normes inhérentes à tout phénomène ou processus comporte un élément nuisible pour tout ce qui entre en contact avec lui (contagion, tant au sens d'infection, que d'imitation psychique, avec toutes les conséquences néfastes que cela entrâine) : dérèglement qu'il subit lui-même lors du contact immédiat, au niveau de la texture par exemple, lorsque la peau entre en contact avec le visqueux et le sale, plus généralement avec la plupart des choses 
nuisibles à l'organisme, etc. Ce n'est pas un hasard si l'expression du dégoût (voir les dessins chez Darwin) se compose d'un mouvement de recul allant dans le sens opposé au stimulus, et laissant à l'"l'arrière-garde" les réactions de défense: rictus découvrant les dents, lèvre inférieure restant légèrement en arrière par rapport au mouvement général et, par conséquent, tendue en avant, organe olfactif se déplaçant non seulement de côté mais vers le haut pour accroître la distance. [La lèvre inférieure] se tord en avant au maximum, se préparant ainsi complètement à l'émission de salive, en tant qu'acte défensif ou mobilisateurréparateur, comme par exemple dans la tendance à laver par la salive une transgression affectant une surface - simple vestige symbolique du rejet primitif par la bouche de ce qui a généré le dégoût (moyen qui, tout comme les tremblements convulsifs, est un vestige indiscutable de l'atavisme qui voulait qu'on se secoue pour se débarrasser de ce qui a déréglé le cours du processus naturel ou qui le menace à une époque où le dégoût psychique n'était pas encore distinct de l'irritation directement physique). Le schéma mécanique de l'expression mimique qui s'oppose structurellement [à ce dégoût] consiste en un rictus agressif - cas de l'attaque interrompue opposé ici au cas de la fuite interrompue ${ }^{V I I}$. [Ces deux cas] marquent un mouvement de rapprochement [par rapport à l'élément irritant] par un paroxysme du dégoût que nous ne détaillerons pas ici (retenons simplement qu'y figure la nausée évoquée ci-dessus).

Par ailleurs, le dégoût est une des expressions apparues au contact immédiat avec la nourriture.

Ce qui provoque le dégoût [l'est] en raison de son anormalité (qui est en soi évidemment relative car la notion d'anormalité dans l'absolu ne peut pas exister), autrement dit dans une situation donnée est alogique, et, conséquemment, provoque le dégoût, alors qu'en soi, i.e. dans d'autres circonstances, il serait parfaitement inoffensif.

Prenons un cas particulier: le fait de se souiller de nourriture (qui constitue ce qu'il y a de plus dégoûtant dans $L a$ Course) se révèle parfaitement normal pour l'enfant dont l'individualité n'est pas encore formée, i.e. qui non seulement n'a pas encore déterminé ses rapports subjectifs avec le monde environnant, 
mais ne s'est pas encore adapté à ses conditions, et en particulier à la nourriture. Dans ces conditions, le même fait est perçu comme parfaitement logique, plus même -- comme utile, en aiguisant l'attention et l'intérêt déjà éveillés de l'enfant à l'égard de ses propres manifestations animales qui ici ne revêtent pas le caractère d'une "curiosité malsaine", mais de quelque chose de parfaitement "sensé", car c'est seulement à travers elles que l'enfant apprend en fin de compte à maîtriser ces manifestations et, en les régulant, effectue ses premiers pas vers l'autodétermination.

La névrose est la conséquence de manifestations trop tardives de traits qui caractérisent la phase infantile et qui lui sont parfaitement naturels et utiles (succion, etc.), mais qui, à l'âge adulte, deviennent inconscients, inutiles, et finalement honteux pour celui qui semble ne pas vouloir se séparer de la tétine de l'enfance, âge d'or de l'irresponsabilité. La "honte» tient ici certes avant tout à l'élément asocial caché, mais tout autant à la personnalité psychique qui y est associée et qui est assez peu ragoûtante en l'absence du processus de sublimation, [sans mentionner] le matériau répugnant qui sert à la satisfaction de ce principe infantile, dans le domaine de l'érotisme (les dites perversions) ou de la production professionnelle du patient.

Comme exemple frappant de la relativité (fondée sur le sentiment intuitif de ce que je viens de développer), de l'effet répulsif d'un même phénomène, je me référerai à la différence d'impression que produisent les innombrables gueules tartinées de chocolat, de chantilly (voire même de pastèque dans le cas du petit nègre, partenaire microscopique de Fatty dans Le Shérif amoureux ${ }^{38}$ ) des gosses de l'écran (tous ces Page, Wesley Barry, etc. et jusqu'à mon petit Michka de $L a$ Grève), par comparaison avec le lumpen* prolétariat galeux de Room qui se barbouille des tomates dont il se goinfre.

Comme on le voit, le répulsif, tant par la force immédiate de l'impression produite qu'en raison de son appartenance aux instincts primaires, les plus profondément enracinés, proches de la tâche essentielle de préservation de l'individu qui donne en définitive son sens au dégoût, est un des moyens les plus forts de produire un effet (d'attractions, c'est-à-dire de phénomènes 
dont les réactions, les réflexes, qu'ils produisent sont connus d'avance). Et Room qui avait pour tâche de provoquer le dégoût pour l'alcool ${ }^{39}$ aurait dû tout naturellement introduire dans son acte sublimatoire d'agitation ce matériau porteur d'attractions qui, de plus, présentait pour lui personnellement un attrait lié à sa névrose.

Ce choix du moyen n'a en soi rien de criminel, car ce qui est décisif, c'est la question du traitement de ce matériau, autrement dit la question de la création de la forme, bien que le fait d'opter pour un matériau de ce type comporte un énorme risque.

Car l'incohérence, l'absence d'unité (notamment des textures et tissus) qui le caractérisent objectivement (dans son "instabilité», manière "d'infantilisme», de stade dépassé, "dans le matériel ») est la pire ennemie et se révèle fatale dans les questions de photogénie, définie comme unité absolue dans la manifestation du principe organisateur, dans la mise en forme intégrale du matériau mort ou des manifestations vivantes ("caractère" pour les questions de physionomie, "profession" pour le mouvement de travail, "construction logique" pour les objets techniques, "logique de la texture" pour les surfaces des objets — fourrure, écaille, poil, etc.).

Et effectivement à cet égard - sous l'aspect photogénique La Course se révèle un échec total. Justement en raison des craintes exprimées ci-dessus, et non parce que le matériau choisi serait russe, tiré du quotidien, et donc "non photogénique": nous avons heureusement sur notre matériau "ingrat" suffisamment d'expériences de découvertes de richesses photogéniques immenses et complètement nouvelles.

Mais ce n'est pas encore le pire - nous sommes toujours prêts à reconnaître la supériorité d'une œuvre correctement réalisée du point de vue de l'agitation qui souffrirait de lapsus formels primitifs, sur la brillance festive d'une photogénie privée d'utilité pour nous.

Je pense même qu'une œuvre conçue avec fougue et assenée [au public] — or toute ouvre d'agitation est avant tout un acte passionnel violent - comporte presque inévitablement des défaillances à un des niveaux formels, et qu'une réussite excessive 
et intégrale [skvoznoe] dans ce domaine «assèche» la perception. La raison en est peut-être que la perception est détournée du dynamisme fougueux du Sturm und Drang vers la statique épique d'une forme classique parfaite, techniquement irréprochable.

Sans parler du fait que c'est seulement à condition d'avoir atteint une pleine maturité dans la maîtrise technique de sa fougue qu'un maître parvient toujours à revêtir les coups qu'il porte d'une perfection semblable, sans pour autant affaiblir leur "portée".

Dans la majorité des cas, [cette perfection] se réalise au détriment de cette "portée", ce qui est d'autant plus triste que du point de vue décisif - du point de vue de celui qui perçoit ce pédantisme excessif se réduit souvent à un travail "en pure perte", du fait que l'instance d'évaluation (de jugement) du récepteur est brisée si la fougue de l'œuvre le captive suffisamment. Sous son aspect grossièrement pratique, cela se produit lorsque la perception de la "beauté» du cadre est sacrifiée, par un dosage (réduit au métrage) minimum, au tempo affirmé par le montage.

La suppression du moment d'évaluation, i.e. le fait de retirer au spectateur la possibilité (dans la durée) d'une perception esthétique contemplative au lieu d'un choc, constitue la pierre angulaire d'un art qui rompt avec son inclusion au rang des phénomènes esthétiques (principe passif dans l'art) et qui prétend seulement (!) au titre d'instrument de lutte (principe actif, révolutionnaire dans l'art).

Quoi qu'il en soit, certains exemples de fougue produisent dans certains cas - par exemple les bandes "comiques" (pas les "comédies" stériles) — un effet beaucoup plus puissant bien que l'élaboration "artistique" en soit des plus primitives: relative négligence dans le filmage, dans les décors, dans la mise en scène, dans la photogénie même du matériau filmé* vir ment dans l'éclairage et en tout cas sans aucun doute dans lintrigue - ce qui contribue largement à produire un effet supérieur (peut-être par la présence d'un élément absurde, introduit dans la technique même de la forme, - peut-être -- mais alors cela vaut également pour le dramatique-tragique qui se caractérise lui aussi par la présence de "l'absurde», de l'alogique — les contrastes se 
touchent - voir pour plus de détails $D u$ tragique de K. Derjavine $^{40}$ ).

Un effet supérieur, j'entends, par rapport à la perfection pédante: comparer la sécheresse formelle (relative, s'entend) de Sois ma femme, avec Max Linder, pleine de sophistications dignes d'un esthète, avec Le Beau Valet et Desert Hero, sans parler des primitifs de Chaplin (les seuls à avoir cours chez nous), emplis de maladresse due à leur spontanéité, qui n'a rien à voir avec le high style de la grande comédie pour "public chic", mais qui emporte la conviction par sa sincérité et correspond peut-être davantage à l'absurdité du contenu qu'elle propose ${ }^{41}$. Le cas de Mister West, comédie "frisquette», est à cet égard intéressant, mais le délai légal étant échu, il ne peut plus être entendu par la cour. On trouvera une certaine analogie formelle avec ce qui vient d'être exposé dans la supériorité de la fougue des esquisses lithographiques de Daumier sur les perfections académiques trop léchées d'Ingres. (Ce n'est pas un hasard si Picasso se tourne justement vers Ingres, au moment où sa fougue décroît dans la période de réaction d'après-guerre).

Mais en revenant à La Course, nous constaterons que l'affaire s'avère beaucoup plus grave.

Room, séduit par la proximité et l'abondance d'un matériau pour lui troublant, collecté soi-disant pour des motifs utilitaires, au lieu de le soumettre au processus de sublimation et à la tâche qu'il s'est fixée, a au contraire succombé à la tentation et suivi une autre voie: celle de la satisfaction de sa névrose dans les formes du film qu'il était en train de créer, au lieu de dépasser ce principe névrotique.

Et c'est là que réside la tragédie. C'est cela qui a déterminé l'accumulation excessive (tant qualitative que quantitative) d'un matériau répulsif qui dépasse toutes les normes de perception de ces phénomènes par des sujets ayant un psychisme sain. C'est cela qui caractérise "l'étalage" complaisant et le plaisir de baigner dans ce matériau.

Les visées d'agitation, au plan social, et de sublimation, au plan personnel, qui donnaient un sens à ce jeu si "périlleux» et le domptaient, ont été perdues. Le réalisateur a transformé l'accomplissement d'un devoir personnel (autocuratif, ce qui en fin 


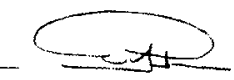

de compte est également socialement utile) et social (d'agitation et d'éducation) en concrétisation de ses propres désirs - en plaisir individuel. Le film est devenu lui-même objet de plaisir, imaginable uniquement pour un psychisme malsain.

Le plaisir produit par ce qui est répugnant est un exemple extrêmement significatif de ce qui est anormal dans la névrose en général: l'utilisation non utilitaire, erronée du dégoût. C'est extrêmement spectaculaire, car n'oublions pas que le dégoût constitue un élément d'autoconservation, le névrosé qui suit la voie de la satisfaction de sa névrose, i.e. de l'utilisation alogique de ses instincts, et donc se dirige vers une mort certaine de sa constitution psychique, se caractérise par là-même comme "atrophiant " l'instinct vital principal d'autoconservation.

Et $\mathrm{La}$ Course s'est mise à provoquer le dégoût, non pas pour la gnôle qu'elle dénonçait, mais pour le film en tant que tel.

Ce dégoût provient non seulement de l'accumulation et de l'étalage indécent d'un matériau répulsif, mais principalement de l'élément asocial que contient le film, clairement perceptible inconsciemment par son association instinctive avec la manifestation sexuelle du même élément, qui ici se produit de façon subtile (quoique moyennement!) - avec l'autosatisfaction du réalisateur (l'onanisme étant la forme d'asocialité la plus tranchée dans les rapports sociaux et sexuels). Ici nous avons affaire à un exemple monstrueux de création d'une œuvre entière, longue de deux mille mètres, visant (pratiquement) uniquement l'autosatisfaction personnelle de l'auteur. Quelque chose comme un peintre qui, pour s'exciter et se satisfaire lui-même, dessinerait des images dans le "genre français». Tout dépend de ce qui passe pour "français" et aux yeux de qui.

En dehors même de l'asocialité consciente, ce genre d'œuvres s'accompagnent inévitablement chez le récepteur de ce sentiment d'hostilité et de répugnance que suscite toujours la représentation publique d'aspects et de manifestations trop personnelles, trop intimes. Il est amusant d'observer le même sentiment de "honte" dans la réception d'un domaine complètement différent - celui du jeu "revivresque » (i.e. lorsque sont sérieusement revécues des situations données), notamment au stade des répétitions, lorsqu'on voit des étalons costauds - des

Rayon et Gnôle [Essai de définition de la carence idéologique dans le domaine de la forme] 


\section{Inédit}

"pros" — se mettre à découvrir naïvement leur âme, et autres côtés intimes! Nous avons ici affaire dans la technique au même cas d'infantilisme - d'introduction dans le jeu forcément conscient de l'adulte de l'élément infantile essentiel du «jeu» de l'enfant - l'inconscient. (Ce qui n'a rien à voir avec [la création] et se trouve, en ce qui concerne la technique de l'acteur, dans un rapport similaire et donne les mêmes résultats que lorsque l'élément infantile intervient dans la constitution d'un adulte normal.) Mais il sera question de cela plus en détail ailleurs.

Une sublimation incomplète qui détermine toute cette mésaventure, telle est la raison première du dégoût éprouvé par tous sans exception (sauf, bien entendu, le patient lui-même, comme il se doit dans les cas de perversion). Cela ne signifie nullement que le matériau lui-même n'y aurait pas contribué par la spécificité de sa tonalité.

L'élaboration de ce genre de matériau, comme de tout matériau "périlleux", équivoque (j’indiquerai à titre d'exemple dans un domaine différent, diverses situations mélodramatiques comme les émotions d'un personnage contraint de tuer son frère, les cas de mort lente et héroïque, etc. - toutes "périlleuses" du point de vue de leur aspect intime, "trou de serrure", du Ekel* [dégoût] qu'elles suscitent à la moindre défaillance de traitement, que la sincérité soit excessive ou le ton complètement faux), afin d'éliminer son "arrière-goût" malsain, demande uniquement un haut degré de maîtrise formelle et d'inventivité employés pour les procédés d'élaboration, autrement dit du traitement, qui consiste à "distiller " le matériau choisi (dosage, confrontation, élaboration rythmique de l'échelle de montage, "pudeur" des prises de vues dans le choix des éléments et des détails qui fixent et dirigent la perception - la formulent, etc.). Dans ce cas, le répulsif en tant que tel (ou le sentimental, ou l'équivoque - dans les questions de traitement, on retrouve les mêmes conditions) ne subsiste que comme présupposé "distillé/purifié", et l'effectivité, déterminée par son empreinte, se déplace de fait sur le "percuteur» formel, construit à partir du présupposé de départ.

Il y a là quelque chose d'analogue, sur le plan formel, à l'élimination de l'élément infantile dans le processus de sublima- 


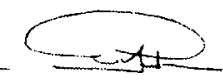

tion. (Je peux me référer aux tableaux 2 et 3 de Moscou, entendstu? mis en scène par moi-même au Premier Théâtre ouvrier comme exemples de solution réussie grâce à leur traitement technique de passages épouvantablement périlleux de par leur aspect mélodramatique.) Bien sûr, cela n'est pensable que comme étape suivante après "distillation » préalable (or de fait, dans l'art, ces processus coïncident, plus exactement l'un apparaît à travers l'autre) de son rôle, soumis névrotiquement au matériau, en rôle assujettissant sublimatoire chez le créateur. Ce que l'on perçoit, c'est le résultat: conviction et recevabilité de ce qui est conscient par opposition à ce qui est honteux - infantile et inconscient.

La Course manque de distillation ${ }^{42}$ !

C'est un enduit non purifié qui "fouette ${ }^{43}$ ", et non un alcool fort qui inflige un coup de fouet en fonction du motif dont il est porteur.

(On pourrait appliquer cela en partie au Rayon où l'on a clairement affaire à des exemples d'hypertrophie de traits fétichistes.)

Les motifs qui déterminent en fait la forme de La Course sont aussi dépourvus d'utilité que dans Le Rayon de la mort, et l'élément de satisfaction se conjugue aussi mal ou plutôt, comme nous le voyons d'après la mécanique du processus, n'est pas combinable (en raison de la différence de ce qui détermine de fait ces motifs) avec les tâches socialement estimables fixées par le réalisateur.

En sociologie, les œuvres dépourvues d'utilité et créées pour d'autres motifs sont qualifiées d'asociales; dans le domaine de l'art - d'esthétisantes. Le caractère esthétisant des principes formels (dans le cas précis «l'art pour l'art»), telle est la plate-forme formelle qui en définitive réunit des œuvres apparemment aussi dissemblables que Le Rayon et La Gnôle (le second cas est particulièrement surprenant dans la mesure où, à la différence du premier, il est fort peu "esthétique »!)

Lappartenance de classe de ce qui est esthétisant nous est assez bien connue et il entrait dans nos objectifs de dévoiler, dans la mesure du possible, cette appartenance sociale des formes, dans les cas examinés, en déterminant les motifs réels de leur mise en forme.

Car seule une prise de conscience précise des principes idéologiques dans des cas existants de formes créées pourra en fin de 
compte nous permettre d'en dévoiler les présupposés et d'instaurer le matérialisme dans les principes de la technique et de la forme, pierre angulaire de l'auto-affirmation d'un art révolutionnaire.

Cet aperçu est une tentative d'utiliser dans le domaine de l'analyse de la forme menée d'un point de vue de classe, une méthode proche de la psychanalyse, considérée comme un des procédés permettant d'établir les présupposés idéologiques.

En fin de compte tous les présupposés socio-économiques déterminant les conditions d'apparition d'une ouvre sont, à eux seuls, insuffisants, [en raison de] la non-correspondance des échelles par rapport à l'analyse de détail d'un point aussi subtil que la forme, surtout lorsqu'il s'agit d'œuvres dont la portée est toute relative.

N'oublions pas en outre que cette analyse, commencée par la névrose, part donc d'un point intermédiaire, sans remonter aux raisons premières de classe de l'émergence de cette névrose, c'est-à-dire à la première moitié du processus, qui ici nous intéressait moins dans la mesure où il présente de l'intérêt pour des recherches dans une autre spécialité que celle que nous analysons.

Nous osons enfin espérer que les «spécialistes" soviétiques cesseront peu à peu, dans leur façon intuitive de "créer", de se borner à n'exprimer dans la forme qu'un contenu idéologique qui leur est propre, en l'introduisant subrepticement comme «emballage" d'un contenu acceptable. Cessant de "créer» à la manière des coqs qui chantent les yeux fermés (comme Ivan Pérestiani qui crée du fond de l'âme), ils essaieront d'aborder de façon consciente cette tâche tout à fait concrète et au fond professionnelle qui est de définir dans chaque cas concret la solution formelle à partir de l'orientation idéologique fondamentale.

Outre le critère de classe, la définition des présupposés individualisants (qui, dans une certaine mesure, sont susceptibles de modification) et des tendances personnelles ne pourra que clarifier le travail et épargner par la suite des déviations. Et que Kouléchov et Room me pardonnent la relative rudesse avec laquelle ces questions ont été soulevées.

Pour le Goskino l'affaire revêt une importance toute particulière dans la mesure où il pourra à l'avenir utiliser rationnelle- 


\section{ment le matériau que constituent les personnalités des réalisa-}

teurs en plaçant "the right man in the right place*".

\section{[NOTES DE SERGUEI EISENSTEIN]}

I. Les organisateurs de pogroms de la RAPP et de $\mathrm{Na}$ postou pensent autrement, mais cela ne concerne que leur conscience privée - en ce qui nous concerne, nous cantonnerons la question à ce piètre endroit?

II. Un de nos jeunes savants psychiatres a posé, de manière très aiguë, la question (dont je ne connais pas les conséquences pratiques) de l'emploi professionnel spécifique par le moyen d'un entraînement spécifique des trait spécifiques qui définissent ladite "défectuosité " d'enfants (en particulier, [les enfants chez qui on décèle] des traits d'insoumission absolue [pourraient être orientés] ultérieurement vers des fonctions de commande et d'initiative dans la suite).

III. Il est évident qu'il peut parfois s'agir d'une impossibilité d'utilisation comme telle. Et c'est là que les organes de direction doivent être encore plus ardemment vigilants. Cela ne concerne pas tant les dirigeants que leur application dans leur travail de préférence pour l'individuel par sa manifestation, tout un système de formes comme une espèce finie (qui correspond plutôt au concept de style, comme les Allemands l'emploient, par exemple, non pas dans la "forme du policier" - in Detectiv-form*, mais dans le style de policier - in Detectiv Stil*) qui ne nous correspond pas idéologiquement "par son origine de classe" et qui existe chez nous, comme une transposition automatique à force de manque de prise de conscience de ce système, soi-disant "neutre ", même "nôtre " à condition de "retournement" de certains indices extérieurs. Entretemps, pour la classe qui avait produit le style donné, il n'est pas qu'un schéma primitif exprimant la classe, qui définit dans le focus particulier les rapports sociaux (et donc le regroupement mécanique de ce qui peut être "soviétisé"), mais dans l'aspect de sa spécificité formelle elle-même il fait partie de l'idéologie de tout un système d'idées de classe, qui influence alors activement celui qui perçoit dans son attitude de classe.

IV. Dans l'alogisme de son déroulement dans la situation donnée - le plus souvent temporaire, plus précisément liée à l'âge, parce que la névrose dans la plupart des cas est déterminée par un résidu infantile.

V. La forme limite de l'effet sadique, l'exécution publique, pouvait dans certains cas - les périodes de la terreur (l'exécution des membres du Narodnä̈a Volia [Volonté du peuple], par exemple) avoir pour tâche et produire, pour peu qu'elle soit accomplie avec suffisamment de logique, exactement l'effet inverse passivant, tandis qu'elle accomplissait avec succès, dans une autre situation, la tâche de susciter l'enthousiasme - par exemple la guillotine.

VI. "De façon artisanale» non par manque de "profondeur", comme il a été dit auparavant, mais par la qualité d'une production consciemment exécutée.

VII. Pour plus de détails sur toute la mécanique des expressions en général, voir la future étude déjà mentionnée sur Les Principes d'organisation des formes et de la technique dans le travail au cinéma, dans la partie traitant du matériau vivant.

VIII. Bien que cela soit ici discutable, mais si cela se produit, c'est en raison d'une curieuse similitude génétique, probablement, du fait lui-même, proposé ici seulement à titre d' "observation", avec l'essence du "comique" en tant que tel.

Traduction du russe: Valérie Posener Notes: François Albera, Valérie Posener, Egor Ovtcharenko 


\section{NOTES DE L'ÉDITEUR}

1. Cet article de 1925 laissé inachevé par Eisenstein se trouve dans les Archives pour l'art et la littérature de la Fédération de Russie (RGALI) qui conservent deux versions de sa première partie (f. 1923, op. 2, ed. 754). Une version légèrement différente, établie par Naoum Kleiman, a été éditée dans Kinoviedchevskie zapiski. Des deux films qu'Eisenstein commente ci-dessous, seul Le Rayon de la mort (1925) de Kouléchov est conservé (sans la dernière bobine) alors que Course à la gnôle (1924) a disparu. On ne dispose plus que de photos du film de Room provenant de l'archive personnelle de $\mathrm{N}$. Lébédev conservée aujourd'hui au Musée du cinéma de Moscou.

2. Ivan Perestjani, auteur des Diablotins rouges (Krasnye d'javoljata), 1923. Ce film passa pour le prototype du film d'aventures bolchévik susceptible de concurrencer la production étrangère. Boris Eikhenbaum lui consacra un article critique dans Jizn' iskusstva, $\mathrm{n}^{\circ} 3,1924$, qui s'achevait ainsi : "Si l'auteur du scénario avait l'intention de divertir les spectateurs, je doute qu'il ait choisi le matériau adéquat. Quant à la propagande, je doute qu'il soir ingénieux de transformer une épopée historique en couplets sur l'air de "Retentis, tonnerre de la victoire!" Cela aussi, c'est assez banal."

3. Spets, néologisme créé après la Révolution pour désigner les spécialistes bourgeois mobilisés au service de la dictature du prolétariat en raison de l'absence de spécialistes "prolétariens". Les spets étaient en général "surveillés" par des " commissaires " prolétariens chargés du contrôle politique de leur travail spécialisé. Le voïenkom était un commissaire militaire chargé de surveiller les spets militaires.

4. La Fin de l'espoir (Op hoop van Zegen) - drame de G. Heiermans situé dans le milieu des pêcheurs hollandais. Dans la Russie d'avant la Révolution, cette pièce était représentée sur les plus grandes scènes de la capitale et de la province. En 1913, le Premier Atelier de MKhAT s'est ouvert avec la représentation de cette pièce (mise en scène de Richard Boleslavski, avec Grigori Khmara, Alexeï Diki, Mikhaill Tchékhov, Sophia Guiatsintova, Séraphima Birman notamment). La pièce est demeurée longtemps après la Révolution au répertoire de plusieurs théâtres d'URSS.

5. MkhAT : acronyme pour Théâtre d'art d'État, le théâtre de Stanislavski.

6. Haut Comité du répertoire.

7. Eisenstein use ici du terme tvortchestvo (création), mor à connotations religieuses qui appartient au discours prémoderne sur l'art. Il l'oppose ici à "mécanique". A la même époque, Kouléchov continue de son côté d'employer ce terme (voir : L. Kouléchov, L'Art du cinéma et autres écrits, Lausanne: L'Âge d'Homme, 1990).

8. Rappelons qu'après $L a$ Grève, Eisenstein travailla en 1924 à un projet d'adaptation au cinéma de Cavalerie rouge d'Isaac Babel, projet abandonné en mars pour L'Année 1905.

9. Jean-Jacques Brousson, Anatole France en pantoufles, Paris: Crès, 1924 (édition russe: 1925). Eisenstein se réfere à nouveau à cet ouvrage dans un texte de 1933, "Le cinéma et la littérature (de l'imagicité) » dans une autre perspective, celle du montage (voir : Le Mouvement de l'art, Paris: Cerf, 1986, p. 31-32).

10. Ce mot formé sur celui de l'écrivain russe Piotr Dmitriévitch Boborykine (18361921) désigne une productivité extraordinaire (plus de 100 romans, nouvelles, pièces de théâtre, livres d'histoire de la littérature, etc.) alliée à une médiocre qualité.

11. Eugen Bleiler (1857-1939), psychiarre et psychologue suisse, qui a étudié la schizophrénie et introduit les concepts de complexe et d'association. Ernst Kretchmer (18881964), médecin psychiatre et psychologue allemand, auteur d'études sur l'hystérie.

12. Cf. les propos de L. Kouléchov sur Fairbanks (notamment "Fairbanks chéri ", 1926, dans: L'Art du cinéma et autres écrits, op. cit.) qui admirait le travail physique, la précision des gestes du Doug, et l'engouement général pour cet acteur dans le public soviétique dont témoigne Le Baiser de Mary Pickford de Komarov. 
13. Léo Mour (Léonid Mourachko, 1889-1938), d'abord administrateur et assistant-réalisateur chez Griffith durant son séjour aux États-Unis avant la révolution. De retour en URSS, il est assistant de L. Kouléchov sur Mister West. Il écrit une série d'articles et de petits livres sur le cinéma américain, intervient à l'ARKK et réalise La Compagnie chaude (1924) et cinq réalisations des années 1925-1928 (pour la plupart non conservées).

14. The Heart of Humanity, Allen Hollubar, 1918, dans lequel Eric von Stroheim est acteur. Le film était imité de celui de Griffith, Hearts of the World.

15. Broken Blossoms, David W. Griffith, 1919.

16. Dimitri Bassalygo (1884-1969), réalisateur au Proletkino et responsable de ce dernier en 1923, tourna des "films d'aventures" sur des thèmes révolutionnaires: Lutte pour l'ultimatum (1923), Arrière-front rouge (1924), De l'́tincelle - la flamme (six épisodes, 1924-1925), La Musulmane (1925), Les Yeux d'Aliocha (1926), Le Voyage de Mr Lloyd (1927), Le Grand et le petit (1929). Il est souvent cité comme repoussoir dans les milieux d'avant-garde (voir: François Albera, "Ejzenstejn e il gruppo Oktjabr", P. Montani (direction), Oltre il Cinema, Venezia: La Biennale, 1991, p. 72).

17. Orphans of the Storm, David W. Griffith, 1921.

18. Way Down Easi, David W. Griffith, 1920.

19. Merry-Go-Round, 1921, d'Eric von Stroheim, sorti en URSS en 1924 sous les titres Adjudant de Franz-Josef et Farce de la vie.

20. Humoresque, 1920, de Frank Borzage, sorti en 1924 en URSS sous les titres $A u$ nom de quoi? et Génie crucifié.

21. Nelly (Nelly, The Beaufiful Cloak Model, 1924), mélodrame du réalisateur américain Emmeth Flynn. Monique Lherbier (La Garçonne) du réalisateur français Armand du Plessy d'après le roman de Victor Margueritte.

22. Foolish Wives, 1922, d'Eric von Stroheim, sorti en URSS sous deux titres: Progéniture de la famille noble et Le Marché aux bassesses. Boris Tomachevski, à l'inverse d'Eisenstein, jugea le film de Stroheim sans intérêt, de "portée idéologique voisine de zéro", relevant d'un "exotisme de pacotille" (Zizn' iskusstva, 1924, $\mathrm{n}^{\circ} 10$, p. 16).

23. Référence au livre d'A. Kachina-Iévréïnova, Le Souterrain du génie (Les sources sexuelles de la création de Dostoïevski), Pétrograd, 1923.

24. Racing Luck, comédie américaine de Herman Reikmaker.

25. Citation approximative de Du Malheur d'avoir de l'esprit de Griboïédov (acte 1, scène 7). Plusieurs passages de cette comédie sont passés au titre de locutions et presque de proverbes en Russie.

26. Sergueï Tretiakov, "Le Théâtre des attractions" in: Dans le front gauche de l'art, Paris: Maspéro, 1977, p. 85-92.

27. Loui-kenz chestnadtsatyi, c'est-à-dire le seizième roi du nom Louis-Quinze.

28. Lacune dans le manuscrit.

29. Dans La Banrière du cinématographe (1920), Kouléchov introduit le terme d'«américanisme" pour désigner le montage et le découpage tels qu'ils se pratiquent dans le cinéma américain. Il le distingue d'une acception plus superficielle, imitative qu'il appelle "l'amerloquisme" (voir L'Art du cinéma et autres écrits, op. cit., p. 38-46 notamment). Le terme est utilisé bien au-delà et en deçà du cinéma depuis la fin $\mathrm{du}$ $\mathrm{XIX}^{\complement}$ siècle, dans le domaine industriel, économique et social.

30. La sortie de Mister West déclenche dans la presse une floraison de considérations sur les chaussures pointues alors symboles de l'Occident (voir: F. Albera, E. Khokhlova, V. Posener, Kouléchov et les siens, Locarno: Festival international du film, 1990).

31. Ce Principes d'organisation dans le travail au cinéma ne figure pas dans les archives d'Eisenstein. On ignore donc s'il a été écrit ou même ébauché. En revanche

Rayon et Gnôle [Essai de définition de la carence idéologique dans le domaine de la forme] 
plusieurs textes sur le mouvement expressif ont été retrouvés qui se fondent sur les théories de Bode et Klages cités plus bas (voir: S. Ejzenstejn, Il Movimento espressivo, Venezia : Marsilio, 1998).

32. Juddy trouvé (Daddy Long Legs, 1919), comédie de Marshall Neilan avec Mary Pickford et Wesley Barry. Sorti en URSS en 1923.

33. Allusions respectivement à Charles Darwin, De l'expression de l'émotion chez l'homme et l'animal (1872) et à Guillaume Benjamin Duchenne, De la physiologie du mouvement (1806-1875).

34. Cette référence à la notion popularisée par Delluc s'explique par la publication de son livre en russe dès 1924 (Fotogenija kino, Moscou: Novye vehi, 1924). Les Formalistes se l'approprient avant de la relativiser - par rapport au montage - (voir : Les Formalistes russes et le cinéma, Paris: Nathan, 1996) et la notion sera discutée dans les revues (voir V. Pertsov, "Le Mythe de la photogénie" dans: Kino-front $\mathrm{n}^{\text {*s }} 2,3 / 5$, 6, 1926).

35. "Pletniev" a la même racine que le verbe russe "plesti" qui signifie ici sous la forme réfléchie "se traîner". Pletniev Fiodorovitch (1886-1942) dramaturge, dirigeant du Proletkult. Eisenstein entra en conflit avec lui en particulier lors du tournage de La Grève, ce qui l'amena à démissionner du mouvement (voir l'interview d'Eisenstein à Kino-nedelya, n' 4, 1925, la lettre ouverte de Pletniev dans le même journal [n" 6, 1925] et la lettre de réponse d'Eisenstein [ ${ }^{\circ}$ 10, 1925] dans: "Falling Out of Proletkult ", Jay Leyda [direction], Eisenstein 2. A Premature Celebration of Eisenstein's Centenary, Calcutta: Seagull Books, 1985, p. 1-8).

36. Dvorec i krepost' (Palais et forteresse) (1924). Réal. : Aleksandr Ivanovskij, scén.: O. Fors, P. Scegolev d'après un roman d'Olga Fors, Vêtement de pierre, image: I. Frolov, V. Glass, interprétation: E. Boronihin, I. Korvin-Krukovskij, E. Hmelevskaja. Le film fut l'objet de beaucoup de controverses dans le milieu du cinéma. Boris Tomachevki et Boris Eikhenbaum lui ont consacré des articles élogieux dans Jizn' iskusstva $\mathrm{n}^{\circ}$ 5, 1926, sous le titre: "Une éclatante victoire de Sevzapkino".

37. Rabkor, littéralement correspondant ouvrier, journaliste qui travaillait en même temps à l'usine en tant qu'ouvrier.

38. Le Shérif amoureux de Fatty (Roscoe Arbuckle) sorti en URSS en 1924.

39. Eau-de-vie non rectifiée, vodka non purifiée produite à l'aide d'un alambic.

40. Nikolaïévitch Derzhavine (1903-1956), historien de littérature, du théâtre et traducteur. En 1922, il publie Du tragique où il analyse la possibilité d'appliquer aux traits principiels et aux circonstances du tragique le principe de la "mécanisation" selon Bergson.

41. Be my Wife, 1921, de Max Linder. Beau Domestique, court métrage comique donné sur les écrans soviétiques en 1923. Fatty dans le désert, titre soviétique de la parodie de Fatty et de Buster Keaton du western Desert Hero, 1919.

42. Gonke nehvataet peregonki: jeu de mot intraduisible entre "Gonka", la course et "peregonka", "surcourse" ou distillation.

43. Jeu de mots: "pue» et "frappe" sont exprimés par le même mot (razit) qui a deux significations différentes. 


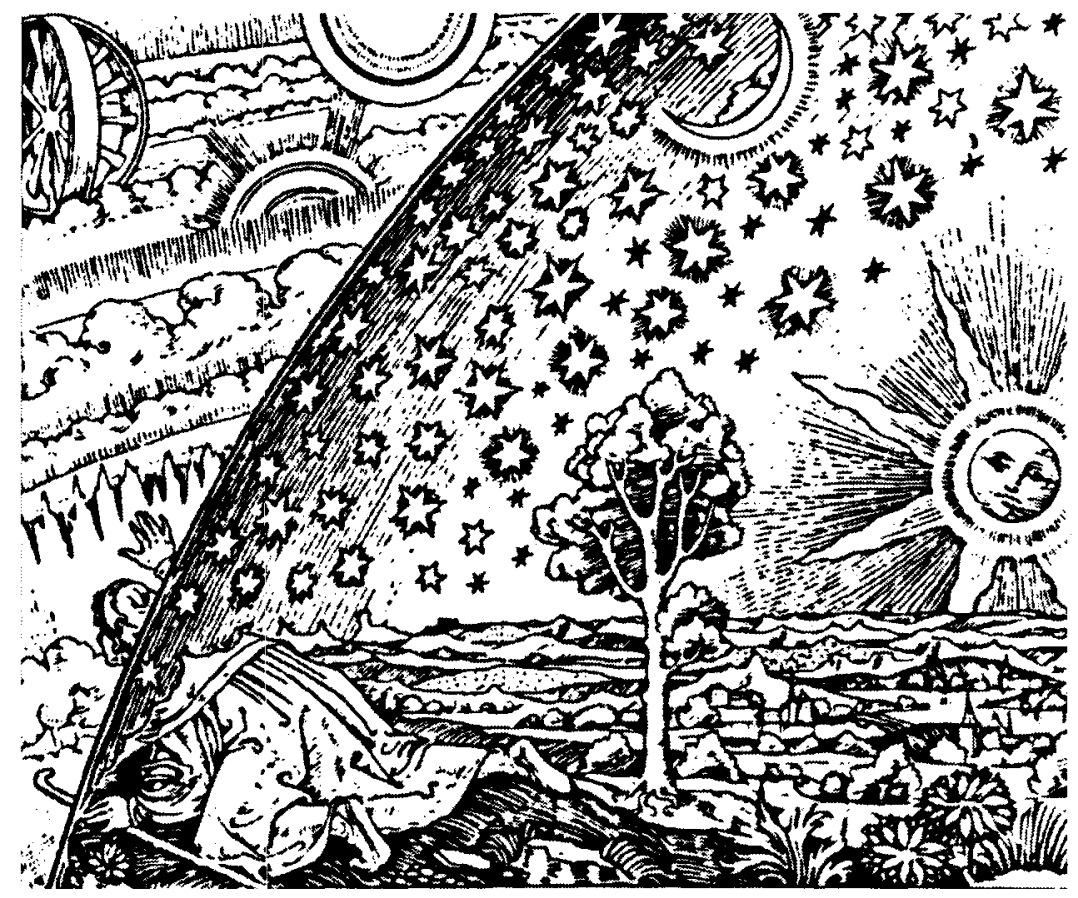

"Dans les problèmes cinématographiques, le film avait jeté un regard “au-delà des étoiles", à l'extérieur de la "sphère des cinéétoiles" dans laquelle gravitaient essentiellement tous les problèmes esthétiques relatifs au cinéma.

Je me sentais comme ce moine, disciple de Nicolas de Cusa, représenté sur une gravure du XVI ${ }^{e}$ siècle: il a passé sa tête avide de savoir de l'autre côté de la sphère des étoiles et ainsi contemple les autres mondes. »

Eisenstein [1940] (1974, p. 109-110). 